\title{
Configuración ideológica y estructura social: resucitando el tema desde la psicología política ${ }^{39}$
}

\author{
Ideological setting and social structure: reviving the \\ topic from the political psychology standpoint
}

Héctor Carvacho ${ }^{40}$ y Andrés Haye A1 $^{41}$

\begin{abstract}
Resumen
A partir de los resultados de una investigación longitudinal sobre la cultura política de tres generaciones de chilenos $\left(n_{1}=494 ; n_{2}=695\right.$; $\left.\mathrm{n}_{3}=494\right)$ se discute la relación entre variables estructurales de la sociedad (generación y clase social) y actitudes relevantes para el posicionamiento ideológico (apoyo a la democracia y autoritarismo). Los resultados indican que entre los jóvenes de derecha el apoyo a la democracia es más alto que entre los adultos de derecha, y que la relación entre autoritarismo y nivel educacional depende de la clase social. Estos resultados sugieren que el estudio actual de la ideología debe volver a tomar en cuenta, además de las variables individuales habitualmente utilizadas en psicología social, la clásica relación entre la estructura social y la formación de las actitudes ideológicas.
\end{abstract}

Palabras clave: ideología; generaciones; clase social; autoritarismo.

\begin{abstract}
In a longitudinal study of the political culture of three generations of Chileans $\left(n_{1}=494 ; n_{2}=695 ; n_{3}=494\right)$ we discuss the relationship between social structural variables (generation and social class) and attitudes concerning their ideological standing (support of de-
\end{abstract}

39 La realización de este trabajo ha sido posible gracias al financiamiento otorgado al proyecto FONDECYT No 1050887 "Estudio Psicosocial de la Cultura Política de Tres Generaciones de Chilenos". En este proyecto participan también los co-investigadores Roberto González, Jorge Manzi y Carolina Segovia. Una versión preliminar de este artículo fue presentada por los autores en los Coloquios de la Escuela de Psicología de la Pontificia Universidad Católica de Chile.

40 Escuela de Psicología de la Pontificia Universidad Católica de Chile. hscarvac@uc.cl

41 Escuela de Psicología de la Pontificia Universidad Católica de Chile. ahaye@uc.cl 
mocracy and authoritarianism). Results show that within young right-wingers support of democracy is higher than within adult right-wingers, and that the relationship between authoritarianism and education level depends on social class. These findings suggest that current research on ideology should take into account, in addition to the individual variables usually considered in social psychology, the old relationship between social structure and the molding of ideological attitudes.

Keywords: ideology; generations; social class; authoritarianism. 


\section{Introducción}

Este artículo presenta una investigación en curso sobre cultura política en la sociedad chilena. Se trata de un estudio longitudinal de 4 años de duración, incluyendo en este transcurso tres mediciones a una misma muestra de santiaguinos. Cada una de estas mediciones es similar en apariencia a una encuesta de opinión pública, pero aborda una serie de temas y conceptos elaborados en la psicología social y la psicología política. Se ha implementado este diseño longitudinal para poder distinguir, a diferencia de lo que ocurre con las encuestas de opinión pública, los aspectos más estables y más hábiles de la cultura política.

En vez de dar cuenta de la investigación en su conjunto, hemos optado por seleccionar una de las hebras de la investigación a modo de ilustración. Específicamente, presentaremos un subconjunto de resultados referidos a la noción de ideología. En particular, resumiremos y discutiremos algunos datos obtenidos en la investigación que problematizan el modo en que tradicionalmente se estudia la ideología desde la psicología social, y que muestran que la configuración ideológica de los chilenos no puede analizarse sin considerar diferencias tanto entre clases sociales como entre grupos generacionales.

\section{La configuración ideológica}

El concepto de ideología, a pesar de ser acuñado originalmente a fines del siglo XVIII por Destut de Tracy para referirse a una "ciencia de las ideas", adquiere relevancia a partir de la obra de Marx y Engels (Jost, Nosek, \& Gosling, 2008). En Marx (Marx \& Engels, 1845/1974), la ideología corresponde tanto a una concepción acerca de la realidad como a una forma de encubrimiento del contexto en que los hombres producen esta realidad. Las formas ideológicas de la conciencia serían entonces la expresión de las relaciones materiales de dominación y permitirían presionar hacia la conservación de las relaciones de poder históricamente condicionadas (Lenk, 1974; Larraín, 2007).

La psicología social ha recurrido a diversos enfoques con el propósito de vincular la producción mental con las relaciones sociales. Entre estos se encuentran el estudio de los conflictos intergrupales (Pratto, Sidanius, \& Levin, 2006; Sidanius, 1985, 1993), de las actitudes (Altmeyer, 1981; 1998), de la identidad grupal (Duckitt, 2001; Stellmacher \& Petzel, 2005), de las representaciones sociales (Scarbrough, 1990), de los estilos cognitivos (Tetlock, 1983), de la cognición social (Jost, Glaser, Kruglansky, \& Sulloway, 2003a, 2003b) y del discurso (Billig, 1984; Billig, Condor, Edwards, Gane, Middleton, \& Radley, 1988). Sin embargo, es difícil encontrar un concepto que reúna de manera teóricamente consistente las distintas formas de abordar la ideología. A En un nivel operacional, usaremos el término "configuración ideológica" para hacer referencia al modo como 
se articulan diversas de actitudes, creencias y valores relativamente estables con que los sujetos pueden posicionarse frente al mundo político en un grupo social determinado. Esta noción supone que habrían actitudes, creencias y valores relativamente estables respecto del mundo político; que tales actitudes, creencias y valores estarían más o menos organizadas entre sí, de tal modo que en diferentes grupos sociales eventualmente podrían estar organizadas de diferente manera; y que esta organización de actitudes, creencias y valores es relevante para entender cómo un sujeto toma posición frente a objetos del mundo político. A pesar de lo discutible de estos supuestos, se trata de una noción consistente con lo expuesto por varios de los más importantes autores en la psicología social para estudiar la ideología (Adorno, Frenkel-Brunswik, Levinson, \& Sanford, 1950; Billig, 1984; Jost, 2006; Kerlinger, 1984).

En la opinión pública, así como en estudios de ciencias políticas y de psicología social, se usa habitualmente el eje izquierda-derecha para resumir la configuración ideológica de los sujetos. El supuesto de esta simplificación es que este eje, de naturaleza intergrupal, estructura la configuración ideológica de manera predominante, pertinente y generalizada. Sin embargo, la utilidad heurística de este eje, como indicador de orientación ideológica de los sujetos, debe ser puesta a prueba en la investigación en cada contexto sociopolítico, pues bien podría ser que la configuración ideológica en un grupo social determinado no esté estructurada por un solo eje predominante o cuya dimensión principal de estructuración no se comporte linealmente.

Si bien en algunos contextos el eje izquierda-derecha puede no ser relevante o bien puede estar perdiendo fuerza en comparación con otras dimensiones emergentes, en varias investigaciones recientes se ha encontrado que este eje sigue resumiendo de la configuración ideológica de los sujetos. Por ejemplo, en el contexto estadounidense, Jost et al. (2003a, 2003b) han identificado dos dimensiones relativamente estables como los ejes de diferenciación ideológica entre liberales y conservadores: (a) actitudes hacia la inequidad y (b) actitudes hacia el cambio social. Estas dos dimensiones, igualitarismo y tradicionalismo, serían acompañadas por otras actitudes más periféricas que variarían en su relevancia dependiendo del momento y del lugar (Jost, 2006). Los sectores liberales y conservadores corresponden, en este contexto, con la izquierda y la derecha, respectivamente. En otro escenario, la investigación que hemos estado desarrollando en Chile, tal como se discutirá más adelante, también confirma que el eje izquierda-derecha permite resumir de modo general la orientación ideológica de los sujetos, pero con contenidos algo diferentes a los que se encuentran en la cultura política estadounidense. En nuestro contexto, y sin variaciones importantes a través de los grupos de edad, el sector de izquierda se asocia sistemáticamente a una mayor valoración de la 
democracia, a menos actitudes de autoritarismo político y a un menor conservadurismo que el sector de derecha.

Ahora bien, el hecho de que el eje izquierda-derecha sea todavía de utilidad heurística en distintos macro-contextos sociopolíticos, no implica que la configuración ideológica sea similar en los distintos contextos. Los resultados que se comentan más adelante se refieren a diferencias encontradas en la configuración ideológica de los sujetos entre contextos sociopolíticos generacionales y de clase social al interior de nuestra sociedad. Las condiciones etáreas y, por tanto, de experiencia histórica de socialización política, así como las condiciones de acceso económico, constituyen determinaciones sociales de la forma de vida de los sujetos, y por tanto tiene sentido analizar más de cerca las configuraciones ideológicas asociadas a estas formas de vida.

\section{El estudio}

Uno de los propósitos centrales de nuestra investigación es caracterizar a tres generaciones de chilenos, socializados políticamente en contextos históricos diferentes. Para ello, hemos definido tres eventuales grupos generacionales en función de rangos de edad de chilenos y chilenas que actualmente son sujetos del mundo político. La generación más antigua está compuesta por aquellas personas que cumplieron 18 años antes del golpe militar, habiéndose socializado durante los gobiernos de Eduardo Frei Montalva y de Salvador Allende. La segunda generación corresponde a personas socializadas políticamente en la segunda mitad de la dictadura, cumpliendo 18 años antes del retorno a la democracia. La tercera generación corresponde a jóvenes que cumplieron 18 años antes de la elección de

Michelle Bachelet, siendo ésta la primera elección presidencial que afrontan con posibilidades de votar, pero cuya socialización política ha trascurrido principalmente dentro de un régimen democrático post-dictatorial.

Así, las tres generaciones se constituyeron a partir de los siguientes grupos etáreos:

- Viejos: 53-63 ańos, cumplieron 18 antes del golpe militar.

- Adultos: 36-43 ańos, cumplieron 18 antes de volver a la democracia.

- Jóvenes: 20-27 años, cumplieron 18 antes de la elección presidencial del 2005.

Además, se distinguieron tres niveles socioeconómicos: alto, medio y bajo con aproximación al siguiente nivel de ingresos (Chile, Ministerio de Planificación, 2006): 
- Alto: ingresos familiares mayores a \$2.000.000 mensuales aproximadamente (correspondiente al 10\% de la población).

- Medio: ingresos familiares entre $\$ 450.000$ y $\$ 2.000 .000$ mensuales aproximadamente (correspondiente al 50\% de la población).

- Bajo: ingresos familiares menores a $\$ 450.000$ mensuales aproximadamente (correspondiente al $40 \%$ de la población).

Se aplicó un muestreo mixto, con selección aleatoria de manzanas del Gran Santiago segmentadas por nivel socioeconómico y asignación de hasta 5 sujetos por manzanas de acuerdo a cuotas por sexo y edad. El contacto se realizó en los domicilios de los sujetos. El grupo socioeconómico alto fue intencionalmente sobrerepresentado para garantizar tamaños muestrales para cada grupo socioeconómico que permitan establecer una comparación intergrupal.

La primera medición, realizada el segundo semestre del 2005, levantó 996 casos válidos, donde el $27 \%$ de los casos corresponde al nivel socioeconómico alto, $37 \%$ al medio, y 36\% al bajo. El 52\% de los casos son mujeres y el 48\% hombres. El 33\% corresponden a la generación mayor, el 33\% a la generación del medio y un $34 \%$ a la generación joven. Esta medición fue hecha 4 meses antes que la primera ronda de la elección presidencial del ańo 2005. La segunda medición fue realizada el segundo semestre del 2006, volviendo a contactar a 695 participantes encuestados el ańo anterior. Adicionalmente se contactó a 146 sujetos para reemplazar la pérdida muestral. La composición muestral es equivalente en términos de niveles socioeconómico, sexo y edad de los participantes. La tercera medición se realizó el primer semestre de 2008 y recontactó a 663 sujetos. De estos, 494 fueron encuestados en las tres mediciones, 73 en la $1^{\text {a }}$ y la $3^{\text {a }}$ medición, y 96 sujetos en la $2^{\mathrm{a}}$ y la $3^{\mathrm{a}}$ medición. La composición muestral también se mantuvo para esta medición.

La pérdida muestral para los sujetos que participaron en todas las mediciones es de 50,4\% entre el año 1 y el 3. Los sujetos que participaron en por lo menos dos mediciones corresponden al 37,2\%, mientras que en solo una medición participó el 13,2\% de los sujetos.

\section{Encuesta}

El instrumento de medición es un cuestionario compuesto por preguntas abiertas y cerradas acerca de distintos ámbitos de la cultura política, tales como: valores democráticos, actitudes políticas (autoritarismo, apoyo a la democracia, conservadurismo, nacionalismo, tolerancia, dominancia social, etc.), otras actitudes sociales (cinismo, alienación, confianza en instituciones, etc.), orientación ideológica y variables sociodemográficas (ingreso y nivel educacional). El cuestio- 
nario fue aplicado por un encuestador entrenado, generalmente en el domicilio de los participantes, tardando entre 45 minutos a una hora su aplicación.

Las preguntas del cuestionario fueron diseñadas para conformar indicadores de constructos que tradicionalmente se utilizan en la psicología social y política para caracterizar la cultura política. De este modo, las preguntas utilizadas son adaptaciones de las escalas más importantes a nivel mundial utilizadas para medir este tipo de constructos, permitiéndonos comparar nuestros resultados con los descritos en publicaciones relevantes en el área.

\section{Variables principales}

El autoritarismo fue medido con una escala de 5 preguntas correspondientes principalmente al componente de agresión autoritaria, identificado como el más importante en la literatura (Altmeyer, 1981, 1998; Funke, 2005). Sus niveles de confiabilidad fueron estables y altos en los tres años (Alpha de Crombach año 1: 0,71, año 2: 0,76 y ańo 3: 0,76).

El apoyo a la democracia fue medido con una escala compuesta por tres preguntas que desagregan la forma en que se pregunta en el Latinobarómetro. Vale decir, en vez de elegir una de las tres alternativas expuestas, se le pide valorar las tres. Su confiabilidad es moderada (Alpha de Crombach año 1: 0,58, año 2: 0,62 y año 3: 0,57).

La orientación ideológica fue medida con una pregunta de auto-posicionamiento en una escala de 1 a 9, donde 1 representa izquierda, 5 representa centro y 9 representa derecha.

El nivel educacional fue indagado de acuerdo a los siguientes niveles: sin educación formal, educación básica incompleta, básica completa, media incompleta, media completa, universitaria incompleta, universitaria completa y universitaria de posgrado. Las personas que tenían educación técnica profesional fueron excluidas de estos análisis para conservar la linealidad de la escala de respuesta.

Para identificar el nivel socioeconómico, además de aproximarse por la segmentación en las manzanas, los encuestadores fueron entrenados en el establecimiento de indicadores que permitieran corroborar la información. Entre estos indicadores se encuentran: el tipo de vivienda, características del barrio, nivel de ingresos, nivel educacional y posesión de bienes. El nivel socioeconómico se presenta como una aproximación a la clase social.

\section{Ideología y actitudes políticas}

Los resultados encontrados muestran que la relación más estrecha entre orientación ideológica y actitudes políticas se da con el autoritarismo y el apoyo a la democracia. Estas actitudes fueron las que presentaron correlaciones más altas 
con orientación ideológica a lo largo de las tres mediciones, superando a todas las otras variables actitudinales que también presentaron una relación significativa: tradicionalismo, temor al desorden, dominancia social (equidad y jerarquía), patriotismo, nacionalismo y cinismo.

Como se ve en la Tabla No 1, esta relación entre orientación ideológica y las dos principales actitudes políticas es estable a lo largo de las mediciones y es de intensidad moderada.

Tabla No 1. Correlaciones ENTRE ORIENTACión IDEOlógiCa Y ACTITUDES POLÍTICAS

\begin{tabular}{|l|c|c|c|}
\hline \multicolumn{1}{|c|}{ Actitudes políticas } & Año I & Año 2 & Año 3 \\
\hline Autoritarismo & $0.28^{*}$ & $0.28^{*}$ & $0.32^{*}$ \\
\hline Apoyo a la democracia & $-0.29^{*}$ & $-0.33^{*}$ & $-0.33^{*}$ \\
\hline
\end{tabular}

* $p<0,0$ I

\section{Actitudes políticas y grupo generacional}

Como puede apreciarse en la Tabla No 2, al segmentar por generaciones las correlaciones se mantienen con un patrón similar para autoritarismo, pero no para apoyo a la democracia, donde se puede apreciar una diferencia importante entre el nivel de apoyo de los jóvenes y de los viejos. Esta diferencia es estable a lo largo de las tres mediciones.

Tabla No 2. CorrelaCiOnES ENTRE ORIENTACIÓN IDEOLÓGICA Y ACTITUDES POLÍTICAS POR GENERACIÓN

\begin{tabular}{|l|c|c|c|c|}
\hline Actitudes políticas & Generación & Año I & Año 2 & Año 3 \\
\hline \multirow{3}{*}{ Autoritarismo } & Jóvenes & $0.20^{*}$ & $0.26^{*}$ & $0.27^{*}$ \\
\cline { 2 - 5 } & Adultos & $0.27^{*}$ & $0.26^{*}$ & $0.36^{*}$ \\
\cline { 2 - 5 } & Viejos & $0.35^{*}$ & $0.31^{*}$ & $0.31^{*}$ \\
\hline \multirow{3}{*}{ Apoyo a la democracia } & Jóvenes & $-0.15^{*}$ & $-0.24^{*}$ & $-0.23^{*}$ \\
\cline { 2 - 5 } & Adultos & $-0.34^{*}$ & $-0.34^{*}$ & $-0.34^{*}$ \\
\cline { 2 - 5 } & Viejos & $-0.37^{*}$ & $-0.39^{*}$ & $-0.41^{*}$ \\
\hline
\end{tabular}

* $p<0,0$ I

\section{Actitudes políticas y clase social}

Por el contrario, al observar la Tabla No 3, puede notarse que el apoyo a la democracia mantiene un patrón de relaciones estable en las tres clases sociales, sin embargo, el autoritarismo presenta diferencias entre la clase social alta y la baja. Estas diferencias son estables a lo largo de las tres mediciones. 
Tabla No 3. CorrelaCiones entre orientaCión ideOlógica y ACTITUDES POLÍTICAS POR CLASE SOCIAL

\begin{tabular}{|l|c|c|c|c|}
\hline Actitudes políticas & Clase & Año I & Año 2 & Año 3 \\
\hline \multirow{4}{*}{ Autoritarismo } & Alta & $0.38^{*}$ & $0.45^{*}$ & $0.47^{*}$ \\
\cline { 2 - 5 } & Media & $0.35^{*}$ & $0.31^{*}$ & $0.41^{*}$ \\
\cline { 2 - 5 } & Baja & $0.23^{*}$ & $0.28^{*}$ & $0.32^{*}$ \\
\hline \multirow{3}{*}{ Apoyo a la democracia } & Alta & $-0.35^{*}$ & $-0.44^{*}$ & $-0.47^{*}$ \\
\cline { 2 - 5 } & Media & $-0.32^{*}$ & $-0.36^{*}$ & $-0.44^{*}$ \\
\cline { 2 - 5 } & Baja & $-0.32^{*}$ & $-0.36^{*}$ & $-0.30^{*}$ \\
\hline
\end{tabular}

* $p<0,01$

Estas dos situaciones invitan a profundizar en los análisis del apoyo a la democracia en las diferentes generaciones, por un lado, y del autoritarismo en las diferentes clases sociales, por otro.

\section{Apoyo a la democracia y generación}

La relación más detallada entre apoyo a la democracia y generación se estudió con un análisis de varianza de mediciones repetidas de la primera variable, incluyendo como variables independientes la orientación ideológica (dividida en tres grupos a partir del auto-posicionamiento en el eje izquierda - derecha) y la generación. Los resultados indican que hay diferencias sistemáticas en función de la orientación ideológica $(F=20,434, p<0,01)$, tal que el apoyo a la democracia es menor mientras el posicionamiento sea más hacia la derecha. Sin embargo, no se observan diferencias según grupo generacional $(F=1,088$, $p>0,05)$. Adicionalmente, y como fenómeno de especial interés, se observa un efecto conjunto de la generación y la orientación ideológica, es decir, una interacción estadísticamente significativa entre las dos variables independientes $(F=3,559, p<0,01)$, de manera que las diferencias en apoyo en función de la orientación ideológica no son homogéneas a través de los grupos de edad. En la Figura 1 se puede apreciar el patrón de esta interacción. El nivel de apoyo a la democracia es más alto en los jóvenes de derecha que en las otras personas de derecha; de hecho, su nivel de apoyo a la democracia es equivalente al de los grupos de centro. Esta diferencia entre los jóvenes y las otras generaciones solo aparece en la derecha. En otras palabras, la observación según la cual el apoyo a la democracia es menor mientras el posicionamiento sea más hacia la derecha, es válida para algunos rangos etarios, y en particular no se cumple en el grupo más joven. Este fenómeno sugiere que para la generación menor el apoyo a la democracia no es un elemento tan relevante en la configuración ideológica, que 
permita diferenciar claramente entre izquierda y derecha. Así, estos datos sugieren que las diferencias en contextos de socialización promueven configuraciones ideológicas diferentes.

\section{Figura No 1. Patrón de apoyo a la democracia por grupo IDEOLÓGICO PARA CADA GENERACIÓN}

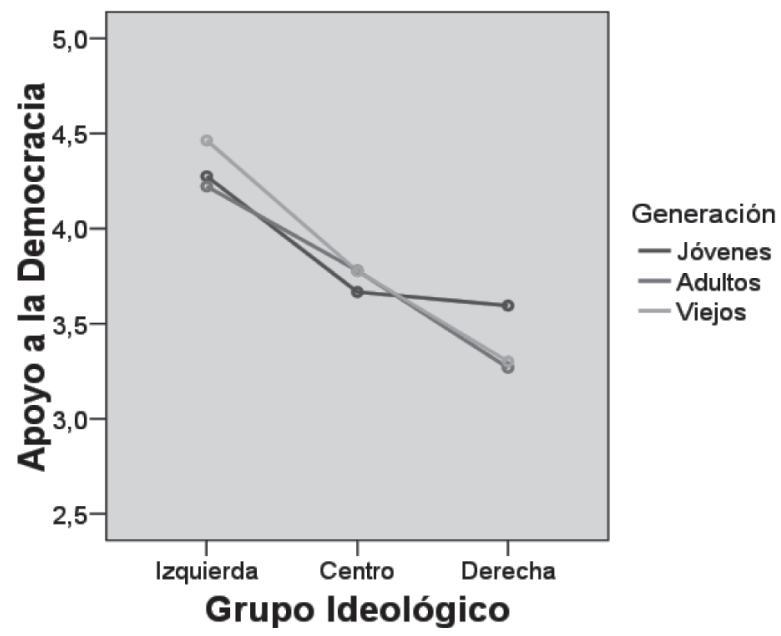

\section{Autoritarismo y clase social}

La relación entre autoritarismo y clase social se exploró inicialmente con un análisis de varianza de mediciones repetidas de la primera vaqriable, empleando como variables independientes la orientación ideológica (3 grupos) y el nivel socioeconómico (como aproximación a la clase social). Los resultados, como puede verse en la Figura № 2, mostraron diferencias sistemáticas en el autoritarismo como función de la orientación ideológica, tal que a mayor derechismo más autoritarismo $(F=26,249, p<0,01)$. Además, se observaron diferencias confiables en función del nivel socioeconómico, tal que a mayor nivel socioeconómico menos autoritarismo $(F=36,832, p<0,01)$. Sin embargo, este efecto del nivel socioeconómico debe ser indagado con mayor detención, pues la correlación de eéste con el nivel educacional es fuerte $(r=0,57)$, y el nivel educacional ha mostrado una relación negativa con el autoritarismo, pues a mayor nivel educacional disminuye el autoritarismo (ver por ejemplo Schuman, Bobo, \& Krysan, 1992). 
Figura No 2. Efectos PRINCIPALES De ORIENTACión IDEOlógica Y Clase en Autoritarismo

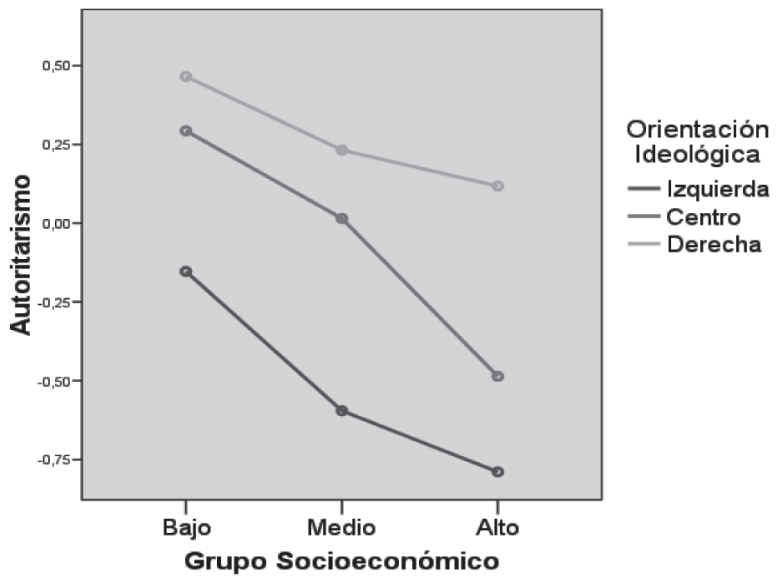

Para distinguir el efecto del nivel socioeconómico del efecto del nivel educacional, se realizó un análisis de varianza de mediciones repetidas de autoritarismo, incluyendo la orientación ideológica y el nivel socioeconómico como variables independientes, y controlando el efecto del nivel educacional incluyendo esta variable como covariante. Los resultados muestran que se mantienen los efectos principales de la orientación ideológica $(F=31,770, p<0,01)$ y el nivel socioeconómico $(F=5,668, p<0,01)$, pero se agrega un efecto de la interacción entre ambas variables $(F=5,559, p<0,01)$. En otras palabras, la observación según la cual el apoyo ael autoritarismo es menor mientras el nivel socioeconómico sea más alto, es cierta solo para quienes se auto-posicionaron como de izquierda o de centro. Los participantes de derecha, por el contrario, mostraron en sus respuestas una tendencia a reflejar altos niveles de autoritarismo independientemente del nivel de acceso económico, manteniendo constantes los niveles de educación.

Como se pueden apreciar en la Figura No 3, el patrón de la interacción muestra que en la clase alta, el efecto de disminución del autoritarismo que tiene el nivel socioeconómico desaparece en el grupo de derecha. Esto indica que la forma en que se relacionan el autoritarismo y la orientación ideológica se diferencia dependiendo del nivel socioeconómico y el nivel educacional, por tanto de la clase social. 
Figura No 3. PATRón de InTERACCIÓn ENTRE ORIENTACIÓN IDEOLÓgica Y CLASE EN AUTORITARISMO CONTROLANDO EL NIVEL EDUCACIONAL

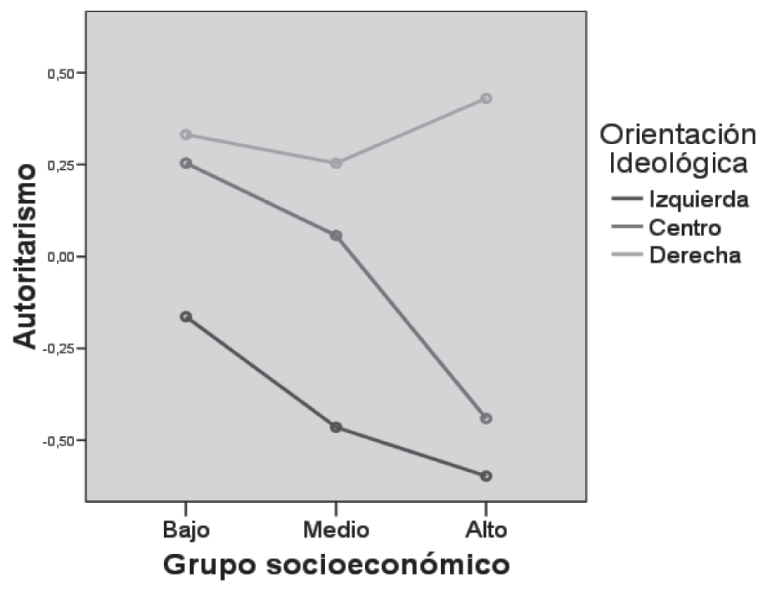

\section{Conclusión}

Las diferencias encontradas entre los jóvenes y los adultos de derecha en sus niveles de apoyo a la democracia, así como la diversa forma en que el autoritarismo se comporta dependiendo de la clase social, indican que la configuración ideológica de los sujetos depende de factores estructurales de la sociedad como clase social y generación. Estas variaciones son esenciales para comprender la diferenciación ideológica en nuestra sociedad, pues en Chile no es lo mismo ser de derecha entre los jóvenes que ser de derecha entre adultos, y la relación entre autoritarismo y nivel educacional no es igual en la clase alta y en la baja.

Los resultados que hemos comentado tienen consecuencias para el estudio de la ideología, problematizando el modo en que se ha abordado tradicionalmente desde la psicología social. Por ejemplo, vinculando la diferenciación ideológica con necesidades psicológicas motivadas por interés personal (Jost, 2006; Jost, Glaser, Kruglansky, \& Sulloway, 2003a, 2003b) o con la necesidad de "justificar el sistema" (Jost, Banaji, \& Nosek, 2004; Jost \& Hunyady, 2002; 2005); que, en último término, serían explicados por aspectos disposicionales como los factores de personalidad "apertura a la experiencia" o "ser concienzudo", o los rasgos de personalidad "necesidad de orden", "necesidad de clausura" o "necesidad de estructura"; y por aspectos situacionales como la amenaza al sistema o el miedo a la muerte (Jost, 2006).

En particular, estos resultados parciales de nuestra investigación muestran que la configuración ideológica de los chilenos no puede analizarse sin considerar 
diferencias entre clases sociales y entre grupos generacionales. Vale decir, la configuración ideológica de las personas debe ser analizada no como un fenómeno mental independiente de la estructura de la sociedad, sino como una configuración de posicionamientos frente al mundo político socialmente "situada".

\section{Bibliografía}

Adorno, T. W., Frenkel-Brunswik, E., Levinson, D., \& Sanford, N. (1950). The Authoritarian Personality. New York: Harper and Row.

Altmeyer, R. A. (1981). Right-wing authoritarianism. Winnipeg, Manitoba, Canada: University of Manitoba Press.

Altmeyer, R. A. (1998). The other "authoritarian personality". Advances in Experimental Social Psychology, 30, 47-91.

Billig, M. (1984). Political ideology: social psychological aspects. En H. Tajfel (Ed.), The social dimension (Vol. 2, págs. 446-470). Cambridge, Great Britain: Cambridge University Press.

Billig, M., Condor, S., Edwards, D., Gane, M., Middleton, D., \& Radley, A. (1988). Ideological Dilemmas. London: Sage Publications.

Chile, Ministerio de Planificación. (2006). Encuesta de Caracterización Socioeconómica Nacional. Santiago: MIDEPLAN.

DuckitT, J. (200I). A dual-process cognitive-motivational theory of ideology and prejudice. Advences in Experimental Social Psychology, 33, 41-113.

Funke, F. (2005). The Dimensionality of Right-Wing Authoritarianism: Lesson from the Dilemma between Theory and Measurement. Political Psychology, $26(2), 195-218$.

Jost, J. T. (2006). The end of the end of ideology. American Psychologist, 61 (7), 651-670.

Jost, J. T., \& Hunyady, O. (2005). Antecedents and consequences of systemjustifying ideologies. Current Directions in Psychological Science, 14, 260 265.

Jost, J. T., \& Hunyady, O. (2002). The psychology of system justification and the palliative function of ideology. European Review of Social Psychology, 13, 111-153.

Jost, J. T., Banaji, M. R., \& Nosek, B. A. (2004). A decade of system justification theory: Accumulated evidence of conscious and unconscious bolstering of the status quo. Political Psychology, 25 (6), 881-919.

Jost, J. T., Glaser, J., Kruglanski, A. W., \& Sulloway, F. (2003b). Exceptions that prove the rule: Using a theory of motivated social cognition to account for ideological incongruities and political anomalies. Psychological Bulletin, 129, 383-393.

Jost, J. T., Glaser, J., Kruglansky, A. W., \& Sulloway, F. (2003a). Political conservatism as motivated social cognition. Psychological Bulletin, 129, 339-375.

Jost, J. T., Nosek, B. A., \& Gosling, S. D. (2008). Ideology. Its Resurgence in Social, Personality, and Political Psychology. Perspectives on Psychological Science, 3 (2), 126-136. 
Kerlinger, F. N. (I984). Liberalism and conservatism: The nature and structure of social attitudes. Hillsdale, NJ: Erlbaum.

Larraín, J. (2007). El Concepto de Ideología (Vol. I: Carlos Marx). Santiago: LOM.

Lenk, K. (1974). El Concepto de Ideología. Buenos Aires: Amorrortu.

MarX, K., \& Engels, F. (1845/1974). La ideología alemana. (W. Roces, Trad.) México: Cultura Popular.

Pratto, F., Sidanius, J., \& Levin, S. (2006). Social dominance theory and the dynamics of intergroup relations: Taking stock and looking forward. European Review of Socila Psychology, 17, 271-320.

Scarbrough, E. (1990). Attitudes, social representations, and ideology. En C. Fraser, \& G. Gaskell (Edits.), The social psychological study of widespread beliefs (págs. 99-117). Oxford: Clarendon Press.

Schuman, H., Bobo, L., \& Krysan, M. (1992). Authoritarianism in the General Population: The Education Interaction Hypothesis. Social Psychology Quarterly, 55 (4), 379-387.

Sidanius, J. (1985). Cognitive functioning and sociopolitical ideology revisited. Political Psychology, 6, 637-661.

Sidanius, J. (1993). The psychology of group conflict and the dynamics of oppression: A social dominance perspective. En S. Iyengar, \& W. McGuire (Edits.), Explorations in political psychology (págs. 183-219). Durham, NC: Duke University Press.

Stellmacher, J., \& Petzel, T. (2005). Authoritarianism as a Group Phenomenon. Political Psychology, 26 (2), 245-274.

Teтьock, P. E. (1983). Cognitive style and political ideology. Journal of Personality and Social Psychology, 45, 118-126.

Fecha de recepción del artículo: 15 de julio de 2008.

Fecha de aceptación del artículo: 24 de noviembre de 2008. 